\title{
Tumor staging in a Beagle dog with concomitant large B-cell lymphoma and T-cell acute lymphoblastic leukemia
}

Journal of Veterinary Diagnostic Investigation 2021, Vol. 33(4) 792-796

(C) 2021 The Author(s)

Article reuse guidelines:

sagepub.com/journals-permissions DOI: $10.1177 / 10406387211011024$ jvdi.sagepub.com

\author{
Alessandro Ferrari, Marzia Cozzi, Luca Aresu, Valeria Martini ${ }^{1}$
}

\begin{abstract}
An 8-y-old spayed female Beagle dog was presented with peripheral lymphadenomegaly. Lymph node cytology and flow cytometry led to the diagnosis of large B-cell lymphoma (LBCL). We detected minimal percentages of LBCL cells in peripheral blood and bone marrow samples. However, a monomorphic population of neoplastic cells different from those found in the lymph node was found in the bone marrow. T-cell acute lymphoblastic leukemia was suspected based on flow cytometric immunophenotyping. PCR for antigen receptor rearrangement (PARR) revealed clonal rearrangement of both B-cell and T-cell receptors, and the presence of both neoplastic clones in the lymph node, peripheral blood, and bone marrow. The dog was treated with multi-agent chemotherapy but died $46 \mathrm{~d}$ following diagnosis. Tumor staging and patient classification are needed to accurately establish a prognosis and select the most appropriate therapeutic protocol.
\end{abstract}

Keywords: acute leukemia; Beagles; flow cytometry; lymphoma; PARR; staging.

An 8-y-old spayed female Beagle dog had occasional cough, lethargy, and weight loss. The dog was treated with nonsteroidal anti-inflammatory drugs to control cough. Given the COVID-19 pandemic in Italy, ${ }^{10}$ the patient could not be evaluated until a month after the first recognition of the clinical signs, at which time peripheral lymphadenomegaly was observed. Nodal cytology was compatible with large cell lymphoma, and the dog was referred to the Clinica Veterinaria San Lorenzo for a second opinion. At admission, generalized lymphadenomegaly, primarily involving mandibular and prescapular lymph nodes (LNs), was observed. The bitch's mucous membranes were slightly pale. Complete blood count $(\mathrm{CBC})$ and blood smear evaluation revealed pancytopenia: moderate, normocytic, normochromic anemia (red blood cells: $3.13 \times 10^{12} / \mathrm{L}$; reference interval [RI]: 5.10 $8.50 \times 10^{12} / \mathrm{L}$ ); severe neutropenia (neutrophils: $0.93 \times 10^{9} / \mathrm{L}$; RI: $3.62-12.3 \times 10^{9} / \mathrm{L}$ ); mild monocytopenia (monocytes: $0.10 \times 10^{9} / \mathrm{L}$; RI: $0.14-1.62 \times 10^{9} / \mathrm{L}$ ); and marked thrombocytopenia (platelets: $39 \times 10^{9} / \mathrm{L}$; RI: $117-490 \times 10^{9} / \mathrm{L}$ ). Routine blood biochemistry results were within RIs. An extremely high C-reactive protein concentration $(>230 \mathrm{mg} / \mathrm{L}$; RI: $<5 \mathrm{mg} / \mathrm{L}$ ) was found. In-house serology for infectious diseases was negative (Theratest quattro; Bioforlife).

Fine-needle aspiration of the right prescapular LN was performed, and cytologic examination repeated. Specimens were stained with May-Grünwald/Giemsa. A predominant population of medium-to-large lymphoid cells, with scant deeply basophilic cytoplasm and a frequent clear perinuclear halo, was seen. The nuclei were round with reticular chromatin and inconspicuous nucleoli (Fig. 1A, 1B). Frequent mitoses were present, as well as occasional small lymphocytes and macrophages with tingible bodies. The diagnosis of lymphoma was confirmed based on these findings.

Abdominal ultrasound examination revealed enlargement of lumbar LNs. Ultrasound-guided cytology of liver and spleen was performed, and both organs were found to be infiltrated by neoplastic cells. No further test was performed on spleen and liver samples.

A second aspirate was collected from the same LN and placed in a tube containing $1 \mathrm{~mL}$ of Roswell Park Memorial Institute medium to perform flow cytometric (FC) immunophenotyping (BriCyte E6; Mindray). Concomitantly, peripheral blood (PB) and bone marrow (BM) samples were collected in EDTA tubes and sent to the FC facility to complete the staging of the disease. FC immunophenotyping was performed within $24 \mathrm{~h}$ of sampling, as described previously. ${ }^{9}$ A dominant population of large cells, positive for CD21, was found in the nodal sample, resulting in a diagnosis of large B-cell lymphoma (LBCL; Fig. 2). The PB contained a few large B cells $(1.03 \%)$; very few large B cells were noted in the $\mathrm{BM}(<1 \%) .{ }^{15}$ Cytologic evaluation of $\mathrm{BM}$ smears revealed a prevalent monomorphic population of mediumto-large round cells with scant-to-moderate clear cytoplasm.

Clinica Veterinaria San Lorenzo, Suisio, Bergamo, Italy (Ferrari); Veterinary Teaching Hospital (Cozzi, Martini), and Department of Veterinary Medicine, University of Milan (Martini), Lodi, Italy; Department of Veterinary Sciences, University of Turin, Grugliasco, TO, Italy (Aresu).

${ }^{1}$ Corresponding author: Valeria Martini, Department of Veterinary Medicine, University of Milan, Via dell'Università 6, Lodi, 26900, Italy. valeria.martini@unimi.it 

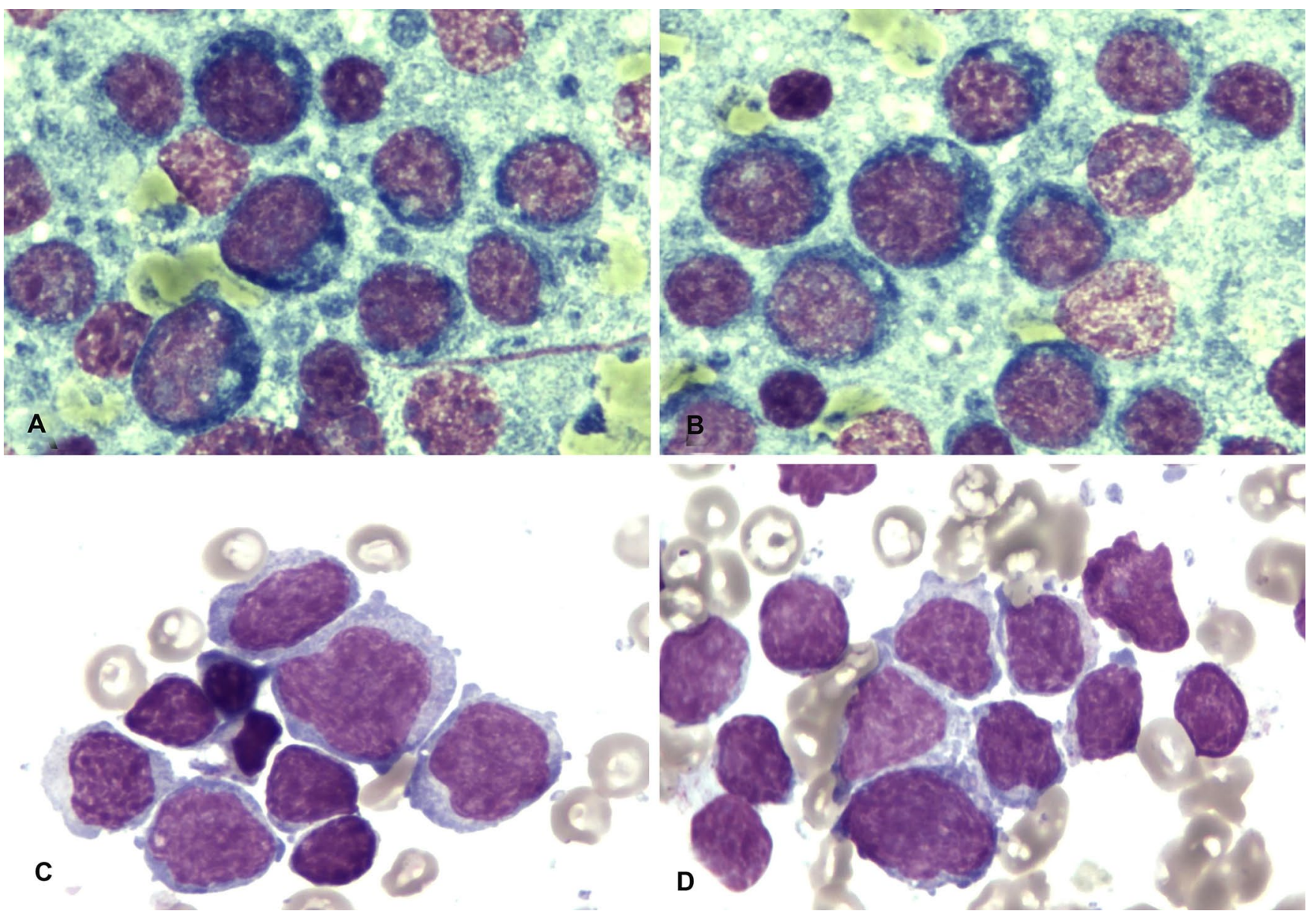

Figure 1. Cytologic findings in a dog diagnosed with large B-cell lymphoma and concomitant acute leukemia of suspected T-cell phenotype. May-Grünwald/Giemsa stain. A, B. Lymph node aspirate, prevalent population of medium-to-large lymphoid cells with basophilic cytoplasm and round nuclei. 100× magnification. C, D. Bone marrow aspirate, prevalent population of large lymphoid cells with clear cytoplasm and irregularly shaped nuclei. 100× magnification.

The nuclei were frequently cleaved and had reticular chromatin; nucleoli were not visible (Fig. 1C, 1D). A few residual hematopoietic cells were present, together with occasional plasma cells and macrophages. The FC antibody panel was extended to the BM sample, and the prevalent population stained positive for panleukocyte markers CD45, CD44, and CD18, but negative for myeloid markers CD14 and CD11b, for the B-cell marker CD21, and for the precursor cell marker CD34. A partial low-positive stain for CD5 (a T-cell marker, 14\%) was detected, but all remaining T-cell markers (CD3, CD4, and CD8) stained negative. The $\mathrm{BM}$ cells also stained negative for MHC II antigen. Based on the morphologic and phenotypic features of the BM cells, acute lymphoblastic leukemia of probable T-cell lineage (T-ALL) was diagnosed (Figs. 1C, 1D, 2F).

PCR for antigen receptor rearrangement analysis (PARR) was performed on the remaining specimens of $\mathrm{LN}$ aspirate, $\mathrm{PB}$, and $\mathrm{BM}$, according to protocols published previously. ${ }^{12}$ Clonal cross-rearrangement of both $\mathrm{B}$ - and T-cell receptors (BCR and TCR) was retrieved on all 3 matrices, and peaks were superimposable (Suppl. Fig. 1). The dog was diagnosed with high-grade LBCL and concomitant suspect T-ALL, with the presence in all matrices of both neoplastic cell types.

The dog was enrolled in a multi-agent chemotherapeutic protocol, including cyclophosphamide, vincristine, and prednisone (COP). ${ }^{4}$ Cytarabine could not be included in the COP protocol because of supply issues linked to the COVID-19 pandemic. Despite initial improvement in the bitch's clinical condition, the LN size remained stable. ${ }^{20}$ After $4 \mathrm{wk}$, a single-agent chemotherapy protocol was implemented with doxorubicin $\left(30 \mathrm{mg} / \mathrm{m}^{2}\right.$ IV). However, the bitch's clinical condition worsened, and euthanasia was elected $46 \mathrm{~d}$ following diagnosis. The owners declined autopsy.

To our knowledge, the concomitant diagnosis of 2 hematopoietic neoplasms, both characterized by aggressive clinical behavior, but involving different lymphocytic lineages, has not been reported previously. A Golden Retriever dog has been reported to have concomitant B-cell lymphoma and 

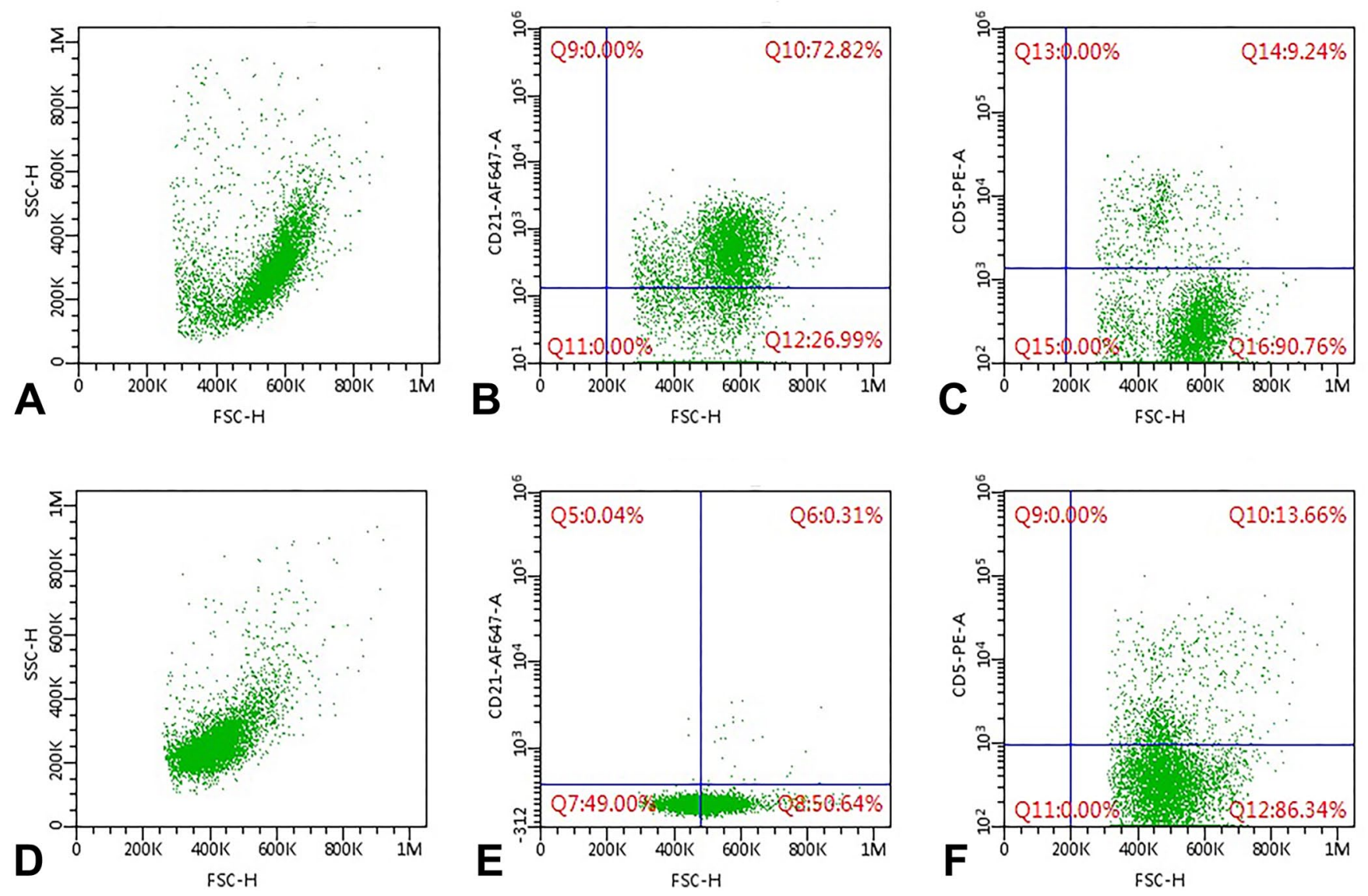

Figure 2. Flow cytometric analysis of a lymph node (LN; A-C) aspirate and a bone marrow (BM; D-F) sample from a dog with concomitant large B-cell lymphoma and acute leukemia of suspected T-cell lineage. Only CD45-positive cells are shown. Discriminators were set based on negative unstained controls. A. Scattergrams of the LN aspirate; cells are displayed based on cell size (FSC-H) and complexity (SSC-H). B. Most of the cells in the LN aspirate were large and stained positive for CD21 (upper right quadrant). C. A few small cells in the LN aspirate stained positive for CD5 (non-neoplastic residual T cells, upper right quadrant). D. Scattergrams of the BM sample; cells are displayed based on cell size (FSC-H) and complexity (SSC-H). E. Large cells staining positive for CD21 represented $<1 \%$ of the cell population in the BM sample (upper right quadrant). F. A subset of neoplastic cells in the BM sample stained positive for CD5 (upper right quadrant).

T-zone lymphoma (TZL). ${ }^{8}$ However, TZL has an indolent clinical course, with prolonged survival even in the absence of therapy, ${ }^{11,17}$ and might have gone unnoticed in the dog until B-cell lymphoma caused clinical signs. Conversely, both LBCL and T-ALL are aggressive diseases, unlikely to remain unnoticed for long periods. Therefore, close onset of both diseases is more probable in our case.

In our case, the diagnosis of lymphoma was made based on nodal cytology within a few days of the visit to the referring clinic. Further characterization of the neoplasm and full staging were undertaken immediately after diagnosis. In the published literature, there is debate regarding the most appropriate workup for dogs with lymphoma, but most clinicians and practitioners limit their diagnostic procedures to cytology alone. ${ }^{14}$ The availability of low-cost advanced diagnostic techniques, requiring minimally invasive sampling (such as FC and PARR), has pushed the scientific community toward better characterization of canine lymphoma, starting with the identification of the lineage of origin., ${ }^{2,18}$ For this patient, we performed FC immunophenotyping of a LN aspirate, revealing a LBCL. Although such a diagnosis can be done confidently by the combined use of cytology and FC, histopathology is required to determine the precise lymphoid neoplasm based on WHO classification. ${ }^{16}$ Unfortunately, in our case, the owners did not consent to a lymphadenectomy or an autopsy.

The usefulness of complete staging for dogs with lymphoma is also debated in the literature. One study demonstrated that inclusion of a larger panel of diagnostic tests resulted in a shift toward higher disease stages in dogs with lymphoma. ${ }^{5}$ However, the official guidelines of the Veterinary Comparative Oncology Group (VCOG) support the analysis of $\mathrm{BM}$ infiltration only in cases of $\mathrm{PB}$ abnormalities. ${ }^{20}$ Accordingly, given that our case was pancytopenic at 
admission, the BM was assessed for neoplastic cell infiltration via FC. A negative result was obtained, suggesting a better prognosis for this patient. ${ }^{9}$ However, BM cytology demonstrated a unique population of neoplastic cells morphologically different from the ones encountered in the $\mathrm{LN}$ aspirate. According to the published literature, deeply basophilic cytoplasm and round nuclei are more commonly encountered in B-cell lymphomas, whereas pale cytoplasm and irregular nuclei are more common in T-cell lymphomas. ${ }^{6,7}$ This observation agrees with our findings in the present case, in which neoplastic cells in the $\mathrm{LN}$ had basophilic cytoplasm and round nuclei and were determined to be of B-cell lineage by FC, whereas those in the BM had clear cytoplasm and indented nuclei and were suspected to be of T-cell lineage, based on FC findings.

To obtain a more detailed picture of our patient, we performed PARR analysis on LN, PB, and BM samples. This diagnostic technique is a reliable tool for confirming a diagnosis of lymphoid malignancy ${ }^{2}$; however, it should be interpreted with caution when assessing the lineage of neoplastic cells, particularly in the case of acute leukemia, given that clonal rearrangement of BCR or TCR is common, even in cases of myeloid neoplasms. ${ }^{19} \mathrm{~A}$ cross-rearrangement of both BCR and TCR was detected in all 3 matrices examined in our case, which could be the result of either the existence of a single neoplastic clone with cross-lineage rearrangement, or to the concomitant presence of 2 different neoplastic clones with lineage-specific rearrangement. This query cannot be solved by PARR alone, but the morphologic appearance and phenotype of the cells support the coexistence of 2 different neoplastic clones in our case. Contrasting results concerning staging were obtained by cytology, FC, and PARR testing. Cross-rearrangement was detected in all 3 matrices by PARR, whereas cytology and FC identified a different, but single, neoplastic population in the LN and BM samples, and no neoplastic cells were seen in the PB sample. These results are likely the result of the higher sensitivity of PARR compared to morphology or morphology plus FC. ${ }^{1,15}$

Based on the presence of acute leukemia, a poor prognosis for this case was given to the owners. However, the owners opted to treat their dog; chemotherapy achieved only a stable disease state. This is a disappointing result given that LBCL in dogs has a response rate of $>90 \%$ for the protocol we used. ${ }^{4}$ The poor response achieved in our patient may be linked to the presence of chemoresistant LBCL cells ${ }^{3}$ or, more probably, to the presence of acute leukemia cells in the LN. Indeed, no effective treatment has been identified for dogs with acute leukemia, although the inclusion of cytosine in the therapeutic protocol has resulted in a moderate increase in survival times. ${ }^{13}$ Unfortunately, cytosine could not be administered in our case.

The diagnosis of concomitant hematopoietic neoplasms in our case was only possible by the full staging procedures adopted after the LBCL diagnosis. An incomplete staging workup would have led to an incomplete diagnosis and prediction of an erroneously much better prognosis for the dog.

\section{Declaration of conflicting interests}

The authors declared no potential conflicts of interest with respect to the research, authorship, and/or publication of this article.

\section{Funding}

The authors received no financial support for the research, authorship, and/or publication of this article.

\section{ORCID iD}

Valeria Martini (iD) https://orcid.org/0000-0002-9882-2867

\section{Supplemental material}

Supplemental material for this article is available online.

\section{References}

1. Aresu L, et al. Minimal residual disease detection by flow cytometry and PARR in lymph node, peripheral blood and bone marrow, following treatment of dogs with diffuse large B-cell lymphoma. Vet J 2014;200:318-324.

2. Burnett RC, et al. Diagnosis of canine lymphoid neoplasia using clonal rearrangements of antigen receptor genes. Vet Pathol 2003;40:32-41.

3. Choi H, et al. Biodynamic digital holography of chemoresistance in a pre-clinical trial of canine B-cell lymphoma. Biomed Opt Express 2018;9:2214-2228.

4. Davies $\mathrm{O}$, et al. Prognostic significance of clinical presentation, induction and rescue treatment in 42 cases of canine centroblastic diffuse large B-cell multicentric lymphoma in the United Kingdom. Vet Comp Oncol 2018;16: 276-287.

5. Flory AB, et al. Stage migration in dogs with lymphoma. J Vet Intern Med 2007;21:1041-1047.

6. Fournel-Fleury C, et al. Cytohistological and immunological classification of canine malignant lymphomas: comparison with human non-Hodgkin's lymphomas. J Comp Pathol 1997; 117:35-59.

7. Fournel-Fleury C, et al. Canine T-cell lymphomas: a morphological, immunological, and clinical study of 46 new cases. Vet Pathol 2002;39:92-109.

8. Long ME, et al. Lymphocytosis and lymphadenopathy in a dog arising from two distinct lymphoid neoplasms. Vet Clin Pathol 2020;49:307-311.

9. Marconato L, et al. Assessment of bone marrow infiltration diagnosed by flow cytometry in canine large B-cell lymphoma: prognostic significance and proposal of a cut-off value. Vet J 2013;197:776-781.

10. Marconato L, Finotello R. Veterinary oncologists adapting to COVID-19 pandemic. Vet Comp Oncol 2020;18:257.

11. Martini V, et al. Canine small clear cell/T-zone lymphoma: clinical presentation and outcome in a retrospective case series. Vet Comp Oncol 2016;14(Suppl 1):117-126.

12. Nicoletti A, et al. CD3-CD20-positive nodal lymphoma with cross-lineage rearrangement in a dog. J Vet Diagn Invest 2020;32:964-967. 
13. Novacco M, et al. Prognostic factors in canine acute leukaemias: a retrospective study. Vet Comp Oncol 2016;14: 409-416.

14. Regan RC, et al. Diagnostic evaluation and treatment recommendations for dogs with substage-a high-grade multicentric lymphoma: result of a survey of veterinarians. Vet Comp Oncol 2013;11:287-295.

15. Riondato F, et al. Analytical and diagnostic validation of a flow cytometric strategy to quantify blood and marrow infiltration in dogs with large B-cell lymphoma. Cytometry B Clin Cytom 2016;90:525-530.

16. Rout ED, Avery PR. Lymphoid neoplasia. Correlations between morphology and flow cytometry. Vet Clin North Am Small Anim Pract 2017;47:53-70.
17. Seelig DM, et al. Canine T-zone lymphoma: unique immunophenotypic features, outcome, and population characteristics. J Vet Intern Med 2014;28:878-886.

18. Sözmen $M$, et al. Use of fine needle aspirates and flow cytometry for the diagnosis, classification, and immunophenotyping of canine lymphomas. J Vet Diagn Invest 2005;17: 323-330.

19. Stokol T, et al. Dogs with acute myeloid leukemia have clonal rearrangements in $\mathrm{T}$ and $\mathrm{B}$ cell receptors. Front Vet Sci 2017;4:76.

20. Vail DM, et al. Response evaluation criteria for peripheral nodal lymphoma in dogs (v1.0) - a Veterinary Cooperative Oncology Group (VCOG) consensus document. Vet Comp Oncol 2010;8:28-37. 